\title{
Aplicação da árvore de decisão fuzzy para análise da conservação de campus universitário privado
}

Marcelo Prado Sucena $\frac{\text { marcelosucena@gmail.com }}{\text { Instituto Militar de Engenharia (IME) - Rio }}$ de Janeiro, Rio de Janeiro, Brasil

Jéssica dos Santos Mota Jsmota1994@gmail.com Universidade Estácio de Sá (UNESA), Rio de Janeiro, Rio de Janeiro, Brasil

Paula Leonardo Silva

\author{
RESUMO
}

Nos dias atuais, a conservação predial é de extrema importância, tendo em vista que as boas condições do ambiente interferem diretamente no dia a dia do local. Com a conservação adequada evitam-se problemas como a depreciação do imóvel, acidentes e problemas estruturais. Este trabalho tem como objetivo desenvolver um estudo de caso na Universidade Estácio de Sá, no Campus Praça XI, aplicando os conceitos das Árvores de Falhas (AF) e de Decisão Fuzzy (ADF) para auxiliar o mapeamento das causas da sua má conservação. Após a estruturação da ADF, será efetuada a análise quantitativa para criar a simulação do cenário atual, além de mais duas simulações para possíveis aplicações de recursos, buscando-se melhores resultados para a conservação do Campus, otimizando-se os recursos. Para se alcançar tal objetivo são realizadas pesquisas aplicada, dedutiva, exploratória, qualitativa e quantitativa com a finalidade de levantar as dados e informações necessários para realizar o estudo de caso. A primeira simulação demonstra a atual condição do Campus, considerando-se situação mais pessimista. As duas outras simulações foram desenvolvidas com o intuito de melhorar a situação atual do Campus, considerando-se melhoria na alocação de recursos. Para primeira análise utiliza-se zero para o grau de pertinência de algumas causas, aquelas consideradas importantes e de solução fácil, mantendo-se a visão pessimista. Na segunda simulação são mantidos os graus de pertinência utilizados na primeira simulação, alterando-se para visão otimista.

PALAVRAS-CHAVE: Conservação. Árvore De Decisão Fuzzy. Árvore De Falhas. Diagrama De Causa E Efeitos. 


\section{INTRODUÇÃO}

$\mathrm{O}$ ato de conservar deveria ser uma prática comum a todos, pois traz diversas vantagens, sendo a principal aumentar a durabilidade e disponibilidade de um produto, serviço que auxiliam e/ou complementam no bem-estar do local.

Segundo o dicionário Aurélio, Conservar nada mais é que manter em bom estado, guardar, preservar, durar, continuar a ter. No mundo em que vivemos hoje, onde as coisas são facilmente descartadas, estamos nos submetendo a um alto consumo e gastos descontrolados, muitas vezes desnecessários, apenas para acompanhar tendências.

A conservação predial se dá em manter as boas condições do ambiente aplicando algumas técnicas. Deve ser planejada de maneira estratégica, pois influencia drasticamente na rotina e desempenho deste local.

Independentemente do tamanho e do ramo faz-se necessária a constante manutenção preventiva dos seus imóveis, para que não haja uma depreciação. Isso pode proporcionar diversos tipos de acidentes, como: diminuição da vida útil do imóvel aumento de problemas estruturais, degradação da imagem da empresa, desvalorização do imóvel e uma influência negativa na produtividade e motivação dos funcionários.

Quando se trata da conservação de um prédio de ensino superior, por ser um local com grande circulação de pessoas, é possível perceber a importância tanto na construção como na preservação e manutenção de sua estrutura. Deve ser adaptável às diferentes circunstâncias, como pessoas com algum tipo de deficiência, idosos e crianças.

Sendo assim, entende-se que a infraestrutura interfere no crescimento de um negócio. Um ambiente que transmita segurança e respeito, que tem o intuito de refletir o aprendizado do aluno faz com que se sinta valorizado, encontrando no Campus a esperança de uma vida melhor.

Pretende-se então desenvolver um estudo de caso pela aplicação da Árvore de Decisão Fuzzy, onde as causas da má conservação de certo Campus Universitário Privado serão decompostas, buscando relacioná-las e analisá-las de forma quantitativa.

$\mathrm{Na}$ sequência serão realizadas simulações considerando possíveis aplicações de recursos, buscando-se oportunidades de melhorias com relação à qualidade e manutenção do ambiente.

Desenvolver-se-ão pesquisas: aplicada, para o desenvolvimento de conhecimento e aplicação prática sobre a conservação do campus universitário; dedutiva, para análise do evento topo, desencadeando as suas possíveis causas para a árvore de decisão; exploratória, para detalhamento dos problemas relacionados à conservação predial, além da construção de hipóteses para simulação; qualitativa, para coleta de informações e detecção das possíveis causas de falhas para a má conservação do campus; quantitativa, para atribuição dos graus de possibilidade de cada causa de falha com relação ao evento topo da árvore de decisão. 


\section{ÁRVORE DE DECISÃO FUZZY (FDT - FUZZY DECISION TREE)}

A FDT é utilizada quando se tem diversas alternativas a serem analisadas para um determinado problema, definindo-o como o Evento Topo, utilizando-se os operadores básicos e os mesmos preceitos da Análise de Árvore de Falhas (FTA - Fault Tree Analysis). $\mathrm{O}$ autor ainda defende a existência de infinitas famílias de união, interseção e complemento e sua importância na combinação dos operadores lógicos OU (or) e/ou E (and) para avaliar de forma geral as diversas alternativas. (XU, 2008, p. 27)

Em Henley e Kumamoto (1981) cita-se que o método da FTA surgiu por volta de 1961, na Bell Telephone Laboratories, desenvolvido por H. A. Watson para suprir a necessidade de avaliar a confiabilidade de um sistema complexo associado ao controle de lançamento de mísseis guiados e de alcance intercontinental.

Para FTA e FDT são utilizados com frequência três operadores fundamentais (and, or e not), de acordo com Scapin (2013). Desta forma, é possível se chegar à probabilidade (no caso da FTA) ou possibilidade (no caso da FDT) de ocorrência do evento topo (ou principal), permitindo-se a compreensão sobre o evento como um todo para análise de segurança e confiabilidade de sistemas complexos.

A análise por FDT, quanto por FTA, baseia-se na construção de um processo lógico e dedutivo de cima para baixo (top-down), onde na parte de cima se tem o evento topo, que é um evento indesejável pré-definido.

Contini (1995) define o Evento Topo como aquele que é anormal no sistema, causado por uma falha, erro humano ou por perturbações. Na parte de baixo da FDT/FTA, após o Evento Topo, apresentam-se os eventos primários, ou seja, as possíveis causas de falhas dele, obtendo-se a visualização clara e fácil da relação entre as falhas. Para se definir a importância de cada falha para o evento topo pode-se utiliza a probabilidade, para a FTA, e a possibilidade Fuzzy (grau de pertinência), para FDT. A FDT e a FTA utilizam símbolos para caracterização, tal como apresentado no quadro 1.

Quadro 1 - Tabela de símbolos

\begin{tabular}{|c|c|}
\hline Símbolos & Funções básicas \\
\hline & E (And) \\
\hline
\end{tabular}

Fonte: Autoria própria (2019) 
Considerando-se os símbolos expostos no quadro 1, Drozda (2013) descreve-os da seguinte forma:

a) Evento Topo: é a combinação das Causas Básicas com os Eventos Intermediários, levando a um evento de saída principal;

b) Eventos Intermediários: é a combinação de uma ou várias Causas Básicas, formando os eventos de saídas;

c) Causa Básica: é definida como a possibilidade para a ocorrência de um Evento Intermediário. Scapin (2007) complementa que ela está no nível mais básico da falha considerado em uma análise de confiabilidade;

d) Porta $\mathrm{E}$ (and): o evento/falha que ocorre somente quando todas as entradas ocorrem de forma simultânea;

e) Porta OU (or): se destaca da porta anterior pela sua operação, pela qual qualquer uma das entradas/eventos (falhas básicas) produz uma resposta.

Baranauskas (2012), explica que os complementos de E e OU como:

a) Não $E$ (Nand): é uma negação ou complemento da porta $E$ que possui as mesmas características operacionais;

b) Não OU (Nor): é uma negação ou complemento da porta OU que possui as mesmas características operacionais.

Segundo Bando et al. (2015) ao elaborar a FDT/FTA para análise de um evento indesejável, utilizando as regras booleanas, é possível analisá-la de forma qualitativa e quantitativa. A análise quantitativa é uma estimativa numérica que determina os parâmetros para as Causas Básicas, Eventos Intermediários e Portas lógicas, definindo a possibilidade (no caso da FDT) de ocorrência do Evento Topo. Este é o foco desse trabalho.

Para estruturação da FDT que balizará a análise quantitativa sustentada pelos preceitos de Yager como exposto em Xu (2008), foram avaliados os procedimentos adotados por Lopes (2016), Espíndola (2015), Wilges (2010), Magri (2009) e o próprio Xu (2008), chegando-se a seguinte metodologia:

a) Coletar informações das falhas que interferem no evento topo a se estudar;

b) Determinar os problemas decorrentes do evento topo, nomeados como Eventos Intermediários;

c) Analisar as falhas e os eventos intermediários, relacionando-os ao evento topo;

d) Analisando-se cada evento intermediário, definir as portas lógicas NOT, OR, AND;

e) Identificar os parâmetros $\mathrm{W}$ de cada porta lógica para se definir a simulação inicial;

f) Definir os graus de possibilidade de cada falha da FDT: aplicar as expressões de Yager para União, Interseção e Complemento, determinando-se o grau de possibilidade de cada evento intermediário e do evento topo. 
Mas, para se tratar da FDT faz-se necessário abordar os preceitos da Teoria Fuzzy que balizarão o entendimento sobre os graus de pertinência ou de possibilidade utilizados na análise quantitativa.

\section{Teoria Fuzzy para FDT}

Segundo Rignel et al. (2011, p.19) a Lógica Fuzzy se iniciou por Jan Lukasiewicz, onde em 1920 ele combinou os conceitos da Lógica Clássica de Aristóteles e introduziu conjuntos com graus de pertinências. Na década de 1960, o professor de Ciências da Computação da Universidade da Califórnia, Lofti Asker Zadeh, se tornou o primeiro autor a fazer uma publicação registrando a sua percepção sobre a Lógica Fuzzy.

De acordo com Gomide et al. (2018) a Lógica Fuzzy baseia-se na Teoria dos Conjuntos Fuzzy, se diferenciando por suas características e detalhes dos Sistemas Lógicos Tradicionais. Os autores afirmam que o uso dessa Lógica proporciona "o raciocínio exato corresponde a um caso limite do raciocínio aproximado, sendo interpretado como um processo de composição de relações nebulosas", ou seja, cada elemento do conjunto Fuzzy, possui um grau de pertinência, o qual pode ser mais próximo da verdade ou não. Na Lógica Fuzzy são utilizados termos linguísticos para caracterizar os valores verdade e são considerados como um subconjunto Fuzzy do intervalo unitário.

Silva (2005) apud Rignel et al. (2011, p. 20) explicam que ao contrário da Lógica Booleana, onde apenas valores booleanos são aceitos, sendo eles definidos como verdadeiros ou falsos, na Lógica Fuzzy os valores variam entre 0 e 1. Assim tem-se que o grau de pertinência, quanto mais próximo ao 1 , mais verdadeiro o valor será; quanto mais próximo a 0 , mais falso o valor será; e se a pertinência permear por 0,5 , então o valor será considerado como talvez verdade.

Os conjuntos Fuzzy podem ser operados seguindo os conceitos de união (or), interseção (and) e complemento (not), supondo A e B como dois conjuntos Fuzzy em um universo de discurso $U$, é possível chegar as seguintes expressões:

$$
\begin{array}{ll}
\text { União (Or) } & (A \cup B)(x)=\max (A(x), B(x))=A(x) \vee B(x) \\
\text { Interseção (And) } & (A \cap B)(x)=\min (A(x), B(x))=A(x) \wedge B(x) \\
\text { Complemento (Not) } & A^{\prime}(x)=1-A(x)
\end{array}
$$

Para a análise das possibilidades de falhas na $\operatorname{FDT} X u(2008$, p. 29) propõe os preceitos de Yager (1980), que utilizam as definições de complemento, união e interseção, conforme as expressões 1, 2 e 3, respectivamente. O autor destaca ainda que o valor da união Yager é maior que a união padrão (max) de Zadeh, ou seja, mais otimista; que o valor de Yager para interseção é menor do que a interseção padrão ( $\mathrm{min}$ ) de Zadeh, isto é, mais pessimista. 


$$
\begin{gathered}
A^{c}(x)=\left(1-A(x)^{w}\right)^{1 / w}, w \in(0, \infty), \\
\left(A \cup_{w} B\right)(x)=\min \left\{1,\left(A(x)^{w}+B(x)^{w}\right)^{\frac{1}{w}}\right\}, w \in(0, \infty), \text { and } \\
\left(A \cap_{w} B\right)(x)=1-\min \left\{1,\left((1-A(x))^{w}+(1-B(x))^{w}\right)^{\frac{1}{w}}\right\}, \\
w \in(0, \infty) .
\end{gathered}
$$

Como se percebe nas expressões 1, 2 e 3, para desenvolvimento da FDT como proposto por $\mathrm{Xu}(2008$, p. 31), apresenta-se ainda o parâmetro $W$ associado ao peso dos operadores lógicos (portas lógicas), tal como identificado nas expressões 4,5 e 6 . 0 valor de $W$ pode ser usado para simulações, pois distingue as causas básicas associadas em uma porta lógica como mais ou menos otimistas/pessimistas.

Para que os pesos sejam definidos como Pessimistas ou Otimistas na FDT é necessário verificar o comportamento do parâmetro $W \in(0, \infty)$. Para interseção ( $\cap)$ o comportamento de $W$ é exposto no Gráfico 1; para união (U) está apresentado o Gráfico 2; e para complemento $\left(A^{\mathrm{C}}\right)$, no Gráfico 3.

Gráfico 1 - Comportamento de W na Interseção de Yager

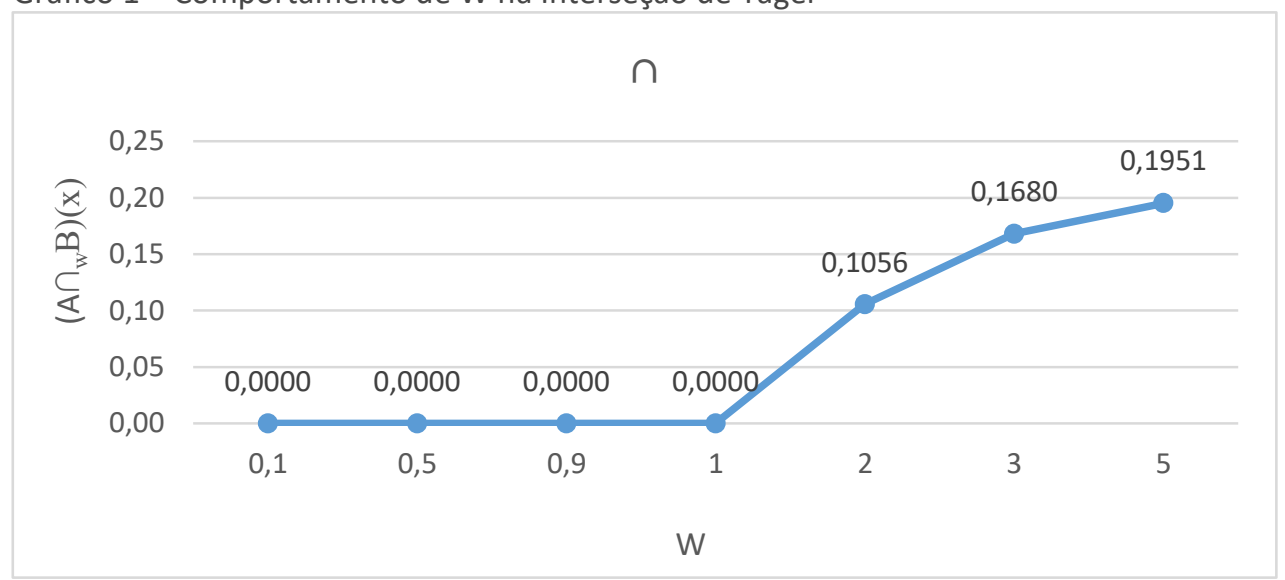

Fonte: Autoria própria (2019)

Gráfico 2 - Comportamento de W na União de Yager

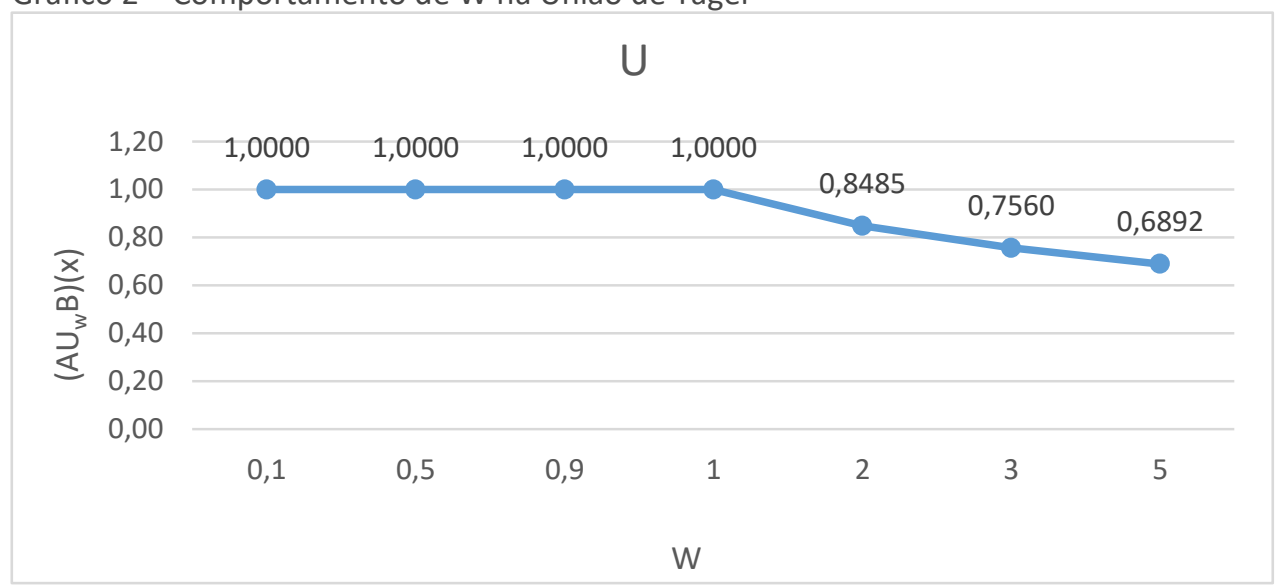

Fonte: Autoria própria (2019) 
Gráfico 3 - Comportamento de W no Complemento de Yager

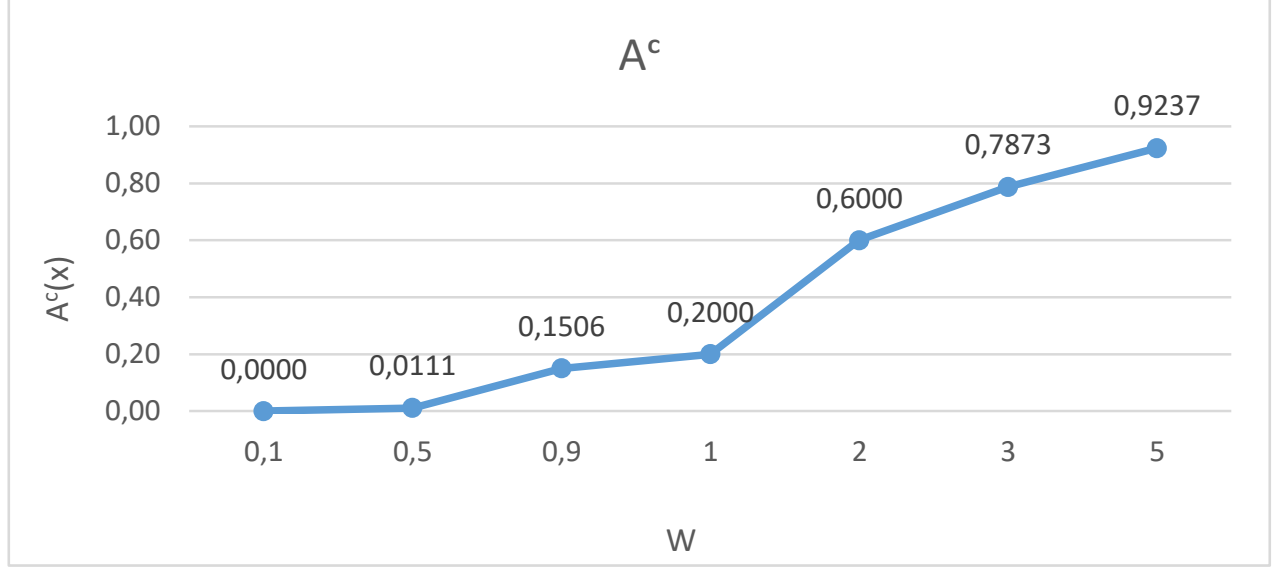

Fonte: Autoria própria (2019)

\section{ESTUDO DE CASO}

\section{ESTRUTURAÇÃO DA ÁRVORE DE DECISÃO FUZZY}

Nesta seção será desenvolvida a estruturação da FDT utilizando como base a metodologia apresentada no item ÁRVORE DE DECISÃO FUZZY (FDT - FUZZY DECISION TREE), segundo os problemas de conservação observados no prédio que abriga o campus universitário, objeto do estudo de caso. Para tanto se faz necessário seguir os seguintes passos:

a) Definir as informações de falhas: foram detectados visualmente diversos problemas na conservação do prédio e na limpeza, refletindo na clara insatisfação dos alunos e professores. As falhas observadas foram: falta aplicação de produto de limpeza, qualidade inadequada dos produtos para higienização, banheiro sujo por falta de ação de funcionário, falta de educação dos usuários, resíduos em excesso nos depósitos, paredes descascadas, rachaduras de pisos e paredes, ventilação inadequada, bebedouro com defeito, cadeiras quebradas, mofo nas paredes e pisos, ar condicionado precário, cadeiras quebradas, falta de material de higiene pessoal nos banheiros, porta dos boxes dos banheiros empenadas e sem trinco e vaso sanitário sem tampa e com danos estruturais;

b) Determinar os eventos intermediários: os eventos intermediários servirão para agrupamento das falhas listadas no item anterior e foram definidos como: limpeza, estrutura e manutenção, que se subdivide em corredores, salas de aula e banheiros;

c) Relacionamento entre os eventos intermediários e as falhas: os eventos intermediários estão vinculados às falhas da seguinte forma:

1 - Limpeza 
1.1 - Falta de produto;

1.2 - Produto de baixa qualidade;

1.3 - Funcionários sem treinamento;

1.4 - Falta de cultura;

1.5 - Coleta de resíduos (lixo): nesse caso utilizar-se-á o complemento (não), pois o que impacta na má conservação do campus é a não coleta de resíduos.

\section{2 - Estrutura}

2.1 - Paredes descascadas;

2.2 - Rachaduras nos pisos e paredes;

2.3 - Falta de ventilação.

3 - Manutenção

3.1 - Corredores

3.1.1- Bebedouros irregulares;

3.1.2 - Cadeiras quebradas.

3.2 - Salas de aula

3.2.1 - Mofo;

3.2.2 - Manutenção precária do ar condicionado;

3.2.3 - Cadeiras irregulares.

\section{3 - Banheiros}

3.3.1 - Irregularidade nos materiais de higiene pessoal;

3.3.2 - Portas empenadas e sem trinco;

3.3.3 - Vasos sanitários irregulares.

Figura 1 - Estrutura da FDT

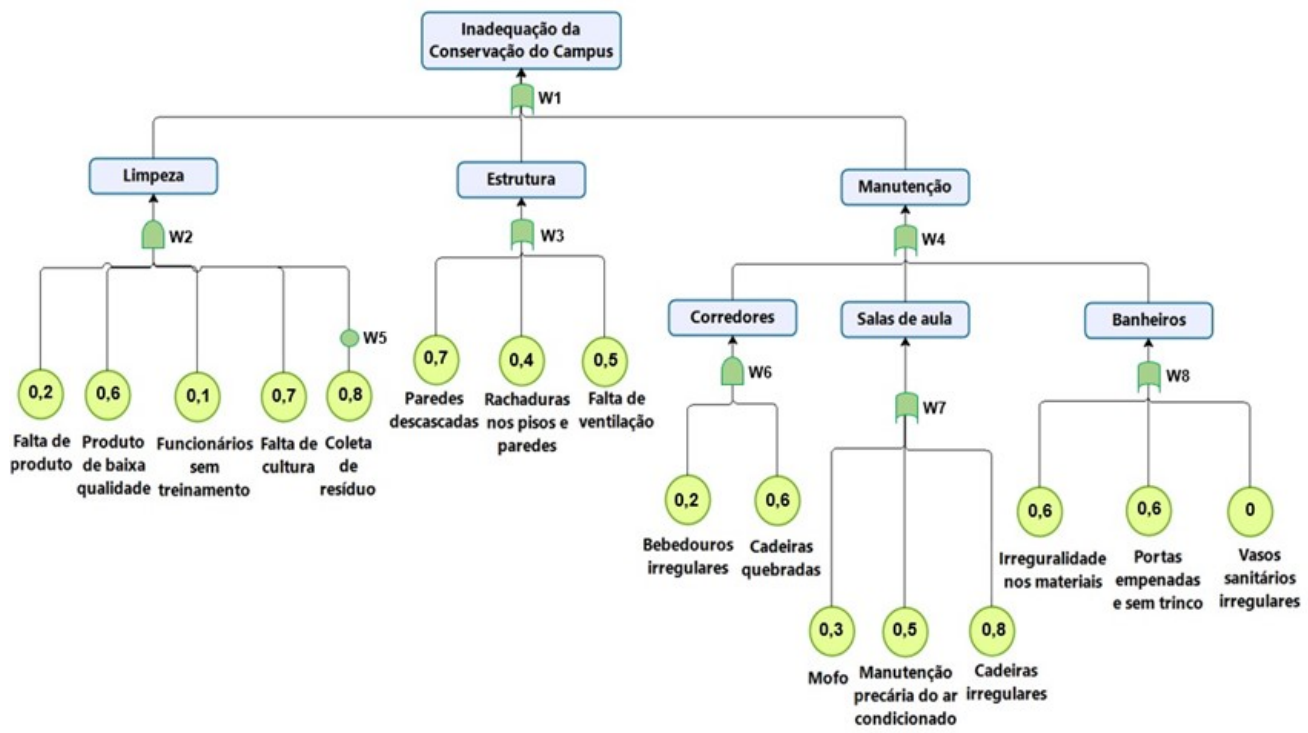

Fonte: Autoria própria (2019) 
d) Definir o Evento Topo: inadequação da conservação do campus;

e) Definir as portas lógicas: com a definição das portas lógicas w1 a w4 é possível visualizar a estrutura da FDT como segue na Figura 1 a seguir;

f) Definir o Evento Topo: inadequação da conservação do campus;

g) Definir as portas lógicas: com a definição das portas lógicas w1 a w4 é possível visualizar a estrutura da FDT como segue na Figura 1 a seguir; Identificação dos valores de W: Considerando-se a análise dos eventos Intermediários da FDT, de forma bottom up, usando como base a Figura 1, definiu-se a importância de cada um, onde os pesos são considerados pessimistas, visto que a situação do campus não está em boas condições.

Para Evento Corredores, definido com a porta $E$ (peso W6) e tendo em vista valores pessimistas quando $W$ é maior que 1 , conforme o Gráfico 1 , estipulou-se valor igual a 1,2, pois circulam muitas pessoas, logo tendo grande visibilidade.

As Salas de Aula, determinadas com a porta OU (peso W7), considerandose valores pessimistas quando W é menor ou igual a 1 , de acordo com o Gráfico 2 , foi determinado valor igual a 0,7 , visto que é onde alunos e professores passam a maior parte do tempo.

Nos Banheiros, definidos com a porta OU (peso W8), considerando-se valores pessimistas, quando $W$ é menor ou igual a 1 , conforme o Gráfico 2, classificou-se com o valor do peso igual a 0,6 , pois a circulação de pessoas é baixa com relação aos demais locais.

Com relação ao Complemento indicado em "Não Coleta de Resíduos" (peso W5), tomando-se valores crescentes de W, eles serão mais pessimistas, levandose em consideração o Gráfico 3 onde foi definido com o peso 0,5, visto que quando essa falha ocorre, impacta diretamente em todos os ambientes do prédio.

Para Limpeza (porta $E$ - peso W2) tem-se valores pessimistas quando W é maior que 1, de acordo com o Gráfico 1, definindo-se com o valor do peso 1,5, já que se faz necessária para que as atividades do prédio aconteçam.

Na Estrutura (porta OU - peso W3) os valores pessimistas para W são considerados quando são menores ou iguais a 1, conforme o Gráfico 2. Estabeleceu-se valor para o peso igual a 0,8 , pois transmite segurança e estabilidade para o ambiente.

Na Manutenção (porta OU - peso W4) consideraram-se valores pessimistas quando $\mathrm{W}$ é menor ou igual a 1 , de acordo com o Gráfico 2, classificado com o peso 0,9 , tomando-se como premissa que sem esta atividade aumenta-se a ocorrência de diversos outros problemas, interferindo na estrutura do local.

Para a porta OU (peso W1), que está na sequência do evento topo, tem-se valores pessimistas quando W é menor ou igual a 1, de acordo com o Gráfico 2 . Considerou-se valor do peso igual a 0,1 , pois a ocorrência reflete diretamente em todos os Eventos Intermediários e Causas Básicas.

Sendo assim, de forma resumida, apresentam-se os pesos das portas lógicas conforme Quadro 2. 
Quadro 2 - Pesos das portas lógicas

\begin{tabular}{|c|c|c|}
\hline \multicolumn{2}{|c|}{ Pesos } & Pessimistas \\
\hline Má conservação do Campus & W1 & 0,1 \\
\hline Limpeza & W2 & 1,5 \\
\hline (Não) Coleta de resíduo & W5 & 0,5 \\
\hline Estrutura & W3 & 0,8 \\
\hline Manutenção & W4 & 0,9 \\
\hline Corredores & W6 & 1,2 \\
\hline Salas de aula & W7 & 0,7 \\
\hline Banheiros & W8 & 0,6 \\
\hline
\end{tabular}

Fonte: Autoria própria (2019)

h) Determinar os graus de possibilidade das falhas: os valores dos graus foram determinados pela observação dos pesquisadores no local, tomando-se que quando há grande possibilidade de acontecer a falha o valor está mais próximo de 1; em contrapartida, quando há pouca possibilidade de acontecer a falha o valor está mais próximo a 0 . Tais valores estão expostos adiante:

1 - Limpeza

1.1 - Falta de produto $-0,2$;

1.2 - Produto de baixa qualidade -0,6;

1.3 - Funcionários sem treinamento - 0,1;

1.4 - Falta de cultura -0,7;

1.5 - Coleta de resíduos (lixo): nesse caso utilizar-se-á o complemento (não), pois o que impacta na má conservação do campus é a não coleta de resíduos $-0,8$.

\section{2 - Estrutura}

2.1 - Paredes descascadas $-0,7$;

2.2 - Rachaduras nos pisos e paredes -0,4;

2.3 - Falta de ventilação -0,5.

3 - Manutenção

3.1 - Corredores

3.1.1 - Bebedouros irregulares - 0,2;

3.1.2 - Cadeiras quebradas -0,6.

3.2 - Salas de aula

3.2.1 - Mofo-0,3;

3.2.2 - Manutenção precária do ar condicionado - 0,5;

3.2.3 - Cadeiras irregulares $-0,8$.

3.3 - Banheiros

3.3.1 - Irregularidade nos materiais de higiene pessoal -0,6;

3.3.2 - Portas empenadas e sem trinco - 0,6;

3.3.3 - Vasos sanitários irregulares - 0. 
Na sequência desta etapa aplicaram as equações de Yager (expressões 4, 5 e 6) para simulação da condição atual do Campus, além de outros dois cenários, a citar.

\section{Simulação Da Situação Atual}

Com o uso da FDT é possível simular para se avaliar a atual situação do campus universitário. A avaliação quantitativa é efetuada na forma bottom up, isto é, das falhas básicas para o evento topo.

Iniciando pelo evento intermediário Manutenção, no ramo Corredores, existe uma porta $\mathrm{E}$ que $\mathrm{W} 6=1,2$, Bebedouros $=0,2$ e Cadeiras quebradas $=0,6$.

Utilizou-se a expressão de Yager para interseção da seguinte forma:

$$
\begin{gathered}
(A \cap B)(x)=1-\min \left\{1 ;\left((1-0,2)^{1,2}+(1-0,6)^{1,2}\right)^{0,83}\right\} \\
(A \cap B)(x)=1-\min \{1 ; 1,08\} \\
(A \cap B)(x)=1-1=0
\end{gathered}
$$

Para o evento intermediário Manutenção, no ramo Salas de Aula, existe uma porta $\mathrm{E}$ com $\mathrm{W} 7=0,7$, com Mofo $=0,3$, Manutenção precária do ar condicionado $=0,5$ e Cadeiras Irregulares $=0,8$. As expressões de Yager para União estão aplicadas adiante.

$$
\begin{gathered}
\left.(A \cup B)(x)=\min \left\{1 ;(0,3)^{0,7}+(0,5)^{0,7}+(0,8)^{0,7}\right)^{1,43}\right\} \\
(A \cup B)(x)=\min \{1 ; 2,52\} \\
(A \cup B)(x)=1
\end{gathered}
$$

Os próximos cálculos estão tabelados a seguir:

Quadro 3-Manutenção de banheiros

\begin{tabular}{|c|c|c|}
\hline \multicolumn{3}{|c|}{ Manutenção } \\
\hline \multicolumn{3}{|c|}{ Banheiros } \\
\hline $\begin{array}{c}\text { Irregularidade nos materiais de } \\
\text { higiene pessoal }\end{array}$ & $\begin{array}{c}\text { Portas empenadas e } \\
\text { sem trinco }\end{array}$ & $\begin{array}{c}\text { Vasos sanitários } \\
\text { irregulares }\end{array}$ \\
\hline 0,6 & 0,6 & 0 \\
\hline
\end{tabular}

Fonte: Autoria própria (2019)

Yager União: $\left.(A \cup B)(x)=\min \left\{1 ;(0,6)^{0,6}+(0,6)^{0,6}+(0)^{0,6}\right)^{1,67}\right\}$

$$
(A \cup B)(x)=\min \{1 ; 1,93\}
$$

$$
(A \cup B)(x)=1
$$

Quadro 4 - Manutanção

\begin{tabular}{|c|c|c|}
\hline \multicolumn{3}{|c|}{ Manutenção W4=0,9 } \\
\hline Corredores & Salas de aula & Banheiros \\
\hline 0 & 1 & 1 \\
\hline
\end{tabular}

Fonte: Autoria própria (2019)

Yager União: $\left.(A \cup B)(x)=\min \left\{1 ;(0)^{0,9}+(1)^{0,9}+(1)^{0,9}\right)^{1,11}\right\}$

$$
\begin{gathered}
(A \cup B)(x)=\min \{1 ; 2,16\} \\
(A \cup B)(x)=1
\end{gathered}
$$


Quadro 5-Estrutura

\begin{tabular}{|c|c|c|}
\hline \multicolumn{2}{|c|}{ Estrutura W3=0,8 } \\
\hline Paredes descascadas & Rachadura nos pisos e paredes & Falta de ventilação \\
\hline 0,7 & 0,4 & 0,5 \\
\hline
\end{tabular}

Fonte: Autoria própria (2019)

Yager União: $\left.(A \cup B)(x)=\min \left\{1 ;(0,7)^{0,8}+(0,4)^{0,8}+(0,5)^{0,8}\right)^{1,25}\right\}$

$(A \cup B)(x)=\min \{1 ; 2,09\}$

$(A \cup B)(x)=1$

Quadro 6 - Limpeza

\begin{tabular}{|c|}
\hline \multicolumn{2}{|c|}{ Limpeza } \\
\hline Complemento $\quad \mathbf{W 5}=\mathbf{0 , 5}$ \\
\hline Coleta de resíduo \\
\hline 0,8 \\
\hline
\end{tabular}

Fonte: Autoria própria (2019)

Yager complemento: $A^{c}(\mathrm{x})=\left(1-0,8^{0,5}\right)^{2}$

$$
A^{c}(\mathrm{x})=0,01
$$

Quadro 7 - Limpeza

\begin{tabular}{|c|c|c|c|c|}
\hline \multicolumn{5}{|c|}{ Limpeza } \\
\hline Falta de produto & $\begin{array}{c}\text { Produto de } \\
\text { baixa qualidade }\end{array}$ & $\begin{array}{c}\text { Funcionários } \\
\text { sem } \\
\text { treinamento }\end{array}$ & $\begin{array}{c}\text { Falta de } \\
\text { cultura }\end{array}$ & $\begin{array}{c}\text { (NÃO) Coleta de } \\
\text { resíduo }\end{array}$ \\
\hline 0,2 & 0,6 & 0,1 & 0,7 & 0,01 \\
\hline
\end{tabular}

Fonte: Autoria própria (2019)

Yager interseção: $\quad(A \cap B)(x)=1-\min \left\{1 ;\left((1-0,2)^{1,5}+(1-\right.\right.$ $\left.\left.0,6)^{1,5}+(1-0,1)^{1,5}+(1-0,7)^{1,5}+(1-0,01)^{1,5}\right)^{0,67}\right\}$

$$
\begin{gathered}
(A \cap B)(x)=1-\min \{1 ; 2,07\} \\
(A \cap B)(x)=1-1=0
\end{gathered}
$$

Quadro 8-Evento Topo

\begin{tabular}{|c|c|c|}
\hline \multicolumn{3}{|c|}{ Evento Topo $\quad$ W1=0,1 } \\
\hline Limpeza & Estrutura & Manutenção \\
\hline 0 & 1 & 1 \\
\hline
\end{tabular}

Fonte: Autoria própria (2019)

$$
\begin{gathered}
\text { Yager União: } \left.(A \cup B)(x)=\min \left\{1 ;(0)^{0,1}+(1)^{0,1}+(1)^{0,1}\right)^{10}\right\} \\
(A \cup B)(x)=\min \{1 ; 1024\} \\
(A \cup B)(x)=1
\end{gathered}
$$

O resultado obtido pelas expressões de Yager para o Evento Topo indica grau de pertinência igual a 1 , ou seja, representa a máxima possibilidade para sua existência, confirmando-se, matematicamente, a percepção de que há má conservação do campus universitário. 


\section{Primeira simulação adicional}

Nesta seção será realizada a simulação, buscando-se resultados mais otimistas para o campus.

Nesta simulação, mantendo-se os pesos $W$ apresentados no quadro 2, serão alterados os graus de pertinência das falhas considerando-se um cenário onde há possível injeção de recursos financeiros e humanos, com o objetivo de mitigar alguns problemas apresentados no campus. O quadro 9 apresenta os graus modificados.

Quadro 9 - Alteração dos graus de pertinência

\begin{tabular}{|c|c|}
\hline Falhas & Graus \\
\hline Falta de produto & 0,2 \\
\hline Funcionários sem treinamento & 0,1 \\
\hline Paredes descascadas & 0,7 \\
\hline Mofo & 0,3 \\
\hline Irregularidade nos materiais de higiene pessoal & 0,6 \\
\hline Portas empenadas e sem trinco & 0,6 \\
\hline
\end{tabular}

Fonte: Autoria própria (2019)

Aplicando-se as expressões de Yager para União (porta OU), Interseção (porta E) e Complemento, utilizando os novos graus pertinência finais, é possível obter os seguintes resultados:

Quadro 10 - Manutenção de corredores

\begin{tabular}{|c|c|}
\hline \multicolumn{2}{|c|}{ Manutenção } \\
\hline Corredores & W6 $=1,2$ \\
\hline Bebedouros irregulares & Cadeiras quebradas \\
\hline 0,2 & 0,6 \\
\hline
\end{tabular}

Fonte: Autoria própria (2019)

$$
\begin{gathered}
\text { Yager } \\
\left.\left.(1-0,6)^{1,2}\right)^{0,83}\right\}
\end{gathered} \text { interseção: } \quad(A \cap B)(x)=1-\min \left\{1 ;\left((1-0,2)^{1,2}+\right.\right.
$$$$
(\mathrm{A} \cap \mathrm{B})(\mathrm{x})=1-\min \{1 ; 1,08\}
$$$$
(A \cap B)(x)=1-1=0
$$

Quadro 11 - Manutenção de salas de aula

\begin{tabular}{|c|c|c|}
\hline \multicolumn{3}{|c|}{ Manutenção } \\
\hline \multicolumn{3}{|c|}{ Salas de aula W7=0,7 } \\
\hline Mofo & Manutenção precária do ar condicionado & Cadeiras irregulares \\
\hline 0 & 0,5 & 0,8 \\
\hline
\end{tabular}

Fonte: Autoria própria (2019)

$$
\begin{gathered}
\text { Yager União: } \left.(A \cup B)(x)=\min \left\{1 ;(0)^{0,7}+(0,5)^{0,7}+(0,8)^{0,7}\right)^{1,43}\right\} \\
(A \cup B)(x)=\min \{1 ; 1,72\} \\
(A \cup B)(x)=1
\end{gathered}
$$


Quadro 12 - Manutenção de banheiros

\begin{tabular}{|c|c|c|}
\hline \multicolumn{3}{|c|}{ Manutenção } \\
\hline \multicolumn{3}{|c|}{ Banheiros } \\
\hline $\begin{array}{c}\text { Irregularidade nos materiais de } \\
\text { higiene pessoal }\end{array}$ & $\begin{array}{c}\text { Portas empenadas e sem } \\
\text { trinco }\end{array}$ & $\begin{array}{c}\text { Vasos sanitários } \\
\text { irregulares }\end{array}$ \\
\hline 0 & 0 & 0 \\
\hline
\end{tabular}

Fonte: Autoria própria (2019)

Yager União: $\left.(A \cup B)(x)=\min \left\{1 ;(0)^{0,6}+(0)^{0,6}+(0)^{0,6}\right)^{1,67}\right\}$

$$
(A \cup B)(x)=\min \{1 ; 0\}
$$

$$
(\mathrm{A} \cup \mathrm{B})(\mathrm{x})=0
$$

Nessa etapa é possível perceber certa alteração no grau de pertinência obtido, denotando-se melhora na Manutenção de Banheiros.

Quadro 13 - Manutenção

\begin{tabular}{|c|c|c|}
\hline \multicolumn{3}{|c|}{ Manutenção } \\
\hline Corredores & Salas de aula & Banheiros \\
\hline 0 & 1 & 0 \\
\hline
\end{tabular}

Fonte: Autoria própria (2019)

$$
\begin{gathered}
\text { Yager União: } \left.(A \cup B)(x)=\min \left\{1 ;(0)^{0,9}+(1)^{0,9}+(0)^{0,9}\right)^{1,11}\right\} \\
(A \cup B)(x)=\min \{1 ; 1\} \\
(A \cup B)(x)=1
\end{gathered}
$$

Quadro 14 - Estrutura

\begin{tabular}{|c|c|c|}
\hline \multicolumn{2}{|c|}{ Estrutura } & \multicolumn{2}{|c|}{ W3 $\mathbf{8}$} \\
\hline Paredes descascadas & $\begin{array}{c}\text { Rachadura nos pisos e } \\
\text { paredes }\end{array}$ & Falta de ventilação \\
\hline 0 & 0,4 & 0,5 \\
\hline
\end{tabular}

Fonte: Autoria própria (2019)

$$
\begin{gathered}
\text { Yager União: } \left.(A \cup B)(x)=\min \left\{1 ;(0)^{0,8}+(0,4)^{0,8}+(0,5)^{0,8}\right)^{1,25}\right\} \\
\qquad \begin{array}{c}
(A \cup B)(x)=\min \{1 ; 1,06\} \\
(A \cup B)(x)=1
\end{array}
\end{gathered}
$$

Quadro 15 - Limpeza coleta de resíduo

\begin{tabular}{|c|}
\hline \multicolumn{2}{|c|}{ Limpeza } \\
\hline Complemento $\quad$ W5 $=0,5$ \\
\hline Coleta de resíduo \\
\hline 0,8 \\
\hline
\end{tabular}

Fonte: Autoria própria (2019) 
Yager complemento: $A^{c}(\mathrm{x})=\left(1-0,8^{0,5}\right)^{2}$

$$
A^{c}(\mathrm{x})=0,01
$$

Quadro 16 - Limpeza

\begin{tabular}{|c|c|c|c|c|}
\hline \multicolumn{4}{|c}{ Limpeza } & W2=1,5 \\
\hline prodta de & $\begin{array}{c}\text { Produto de } \\
\text { baixa } \\
\text { qualidade }\end{array}$ & $\begin{array}{c}\text { Funcionários } \\
\text { sem } \\
\text { treinamento }\end{array}$ & $\begin{array}{c}\text { Falta de } \\
\text { cultura }\end{array}$ & $\begin{array}{c}\text { (NÃO) Coleta de } \\
\text { resíduo }\end{array}$ \\
\hline 0 & 0,6 & 0 & 0,7 & 0,01 \\
\hline
\end{tabular}

Fonte: Autoria própria (2019)

Yager interseção: $(A \cap B)(x)=1-\min \left\{1 ;\left((1-0)^{1,5}+(1-0,6)^{1,5}+\right.\right.$ $\left.\left.(1-0)^{1,5}+(1-0,7)^{1,5}+(1-0,01)^{1,5}\right)^{0,67}\right\}$

$$
\begin{gathered}
(A \cap B)(x)=1-\min \{1 ; 2,26\} \\
(A \cap B)(x)=1-1=0
\end{gathered}
$$

Quadro 17 - Má conservação do Campus Praça XI

\begin{tabular}{|c|c|c|}
\hline \multicolumn{2}{|c|}{ Má conservação do Campus Praça XI } & W1=0,1 \\
\hline Limpeza & Estrutura & Manutenção \\
\hline 0 & 1 & 1 \\
\hline
\end{tabular}

Fonte: Autoria própria (2019)

Yager União: $\left.(A \cup B)(x)=\min \left\{1 ;(0)^{0,1}+(1)^{0,1}+(1)^{0,1}\right)^{10}\right\}$

$$
(A \cup B)(x)=\min \{1 ; 1024\}
$$$$
(\mathrm{A} \cup \mathrm{B})(\mathrm{x})=1
$$

O resultado final obtido apresenta grau de pertinência igual a 1 , ou seja, a alteração sugerida no quadro 9 não foi suficiente para melhorar o resultado na Má Conservação do campus, mantendo-se os pesos W como pessimistas.

\section{Segunda simulação adicional}

Na segunda simulação, mantendo-se os graus de pertinência apresentados no quadro 9 , será realizada a alteração nos pesos $W$ de forma otimista. $O$ quadro 18 apresenta os pesos $W$ adotados como otimistas.

Quadro 18 - Alteração dos pesos W

\begin{tabular}{|c|c|c|c|}
\hline \multicolumn{2}{|c|}{ Pesos } & Pessimistas & Otimistas \\
\hline Má conservação do Campus & W1 & 0,1 & 5 \\
\hline Limpeza & W2 & 1,5 & 0,4 \\
\hline (Não) Coleta de resíduo & W5 & 0,5 & 1 \\
\hline Estrutura & W3 & 0,8 & 2,5 \\
\hline Manutenção & W4 & 0,9 & 4 \\
\hline Corredores & W6 & 1,2 & 0,5 \\
\hline
\end{tabular}

Fonte: Autoria própria (2019)

Aplicando-se as expressões de Yager para União (porta OU), Interseção (porta E) e Complemento, utilizando os graus pertinência expostos no quadro $9 \mathrm{e}$ os pesos otimistas do quadro 18, é possível obter os seguintes resultados: 
Quadro 19 - Manutenção - corredores

\begin{tabular}{|c|c|}
\hline \multicolumn{2}{|c|}{ Manutenção } \\
\hline Corredores & W6 $=0,5$ \\
\hline Bebedouros irregulares & Cadeiras quebradas \\
\hline 0,2 & 0,6 \\
\hline
\end{tabular}

Fonte: Autoria própria (2019)

$$
\begin{gathered}
(A \cap B)(x)=1-\min \left\{1 ;\left((1-0,2)^{0,5}+(1-0,6)^{0,5}\right)^{2}\right\} \\
(A \cap B)(x)=1-\min \{1 ; 2,31\} \\
(A \cap B)(x)=1-1=0
\end{gathered}
$$

Quadro 20 - Manutenção - salas de aula

\begin{tabular}{|c|c|c|}
\hline \multicolumn{3}{|c|}{ Manutenção } \\
\hline \multicolumn{3}{|c|}{ Salas de aula } \\
\hline Mofo & Manutenção precária do ar condicionado & Cadeiras irregulares \\
\hline 0 & 0,5 & 0,8 \\
\hline
\end{tabular}

Fonte: Autoria própria (2019)

$$
\begin{gathered}
\text { Yager União: } \left.(A \cup B)(x)=\min \left\{1 ;(0)^{3}+(0,5)^{3}+(0,8)^{3}\right)^{0,33}\right\} \\
(A \cup B)(x)=\min \{1 ; 0,86\} \\
(A \cup B)(x)=0,86
\end{gathered}
$$

Quadro 21 - Manutenção de banheiros

\begin{tabular}{|c|c|c|}
\hline \multicolumn{3}{|c|}{ Manutenção } \\
\hline \multicolumn{2}{|c|}{ Banheiros } & W8=1 \\
\hline $\begin{array}{c}\text { Irregularidade nos materiais } \\
\text { de higiene pessoal }\end{array}$ & $\begin{array}{c}\text { Portas empenadas e sem } \\
\text { trinco }\end{array}$ & $\begin{array}{c}\text { Vasos sanitários } \\
\text { irregulares }\end{array}$ \\
\hline 0 & 0 & 0 \\
\hline
\end{tabular}

Fonte: Autoria própria (2019)

$$
\begin{gathered}
\text { Yager União: } \left.(A \cup B)(x)=\min \left\{1 ;(0)^{1}+(0)^{1}+(0)^{1}\right)^{1}\right\} \\
(A \cup B)(x)=\min \{1 ; 0\} \\
(A \cup B)(x)=0
\end{gathered}
$$

Quadro 22 - Manutenção

\begin{tabular}{|c|c|c|}
\hline \multicolumn{3}{|c|}{ Manutenção } \\
\hline Corredores & Salas de aula & Banheiros \\
\hline 0 & 1 & 0 \\
\hline
\end{tabular}

Fonte: Autoria própria (2019)

Yager União: $\left.(A \cup B)(x)=\min \left\{1 ;(0)^{4}+(0,86)^{4}+(0)^{4}\right)^{0,25}\right\}$

$$
\begin{gathered}
(A \cup B)(x)=\min \{1 ; 0,86\} \\
(A \cup B)(x)=0,86
\end{gathered}
$$

Pode-se identificar alteração no grau de pertinência entre a primeira e segunda simulações adicionais, de 1 para 0,86, ao modificar as Falhas de Manutenção, que são mofo, irregularidade nos materiais de higiene pessoal, 
portas empenadas e sem trinco. Os pesos foram alterados para forma otimista, sendo possível obter uma melhora no resultado final com relação a Manutenção.

Quadro 23 - Estrutura

\begin{tabular}{|c|c|c|}
\hline \multicolumn{2}{|c|}{ Estrutura } & W3=2,5 \\
\hline Paredes descascadas & $\begin{array}{c}\text { Rachadura nos pisos e } \\
\text { paredes }\end{array}$ & 0,5 \\
\hline 0 & 0,4 & Fentilação \\
\hline
\end{tabular}

Fonte: Autoria própria (2019)

Yager União: $\left.(A \cup B)(x)=\min \left\{1 ;(0)^{2,5}+(0,4)^{2,5}+(0,5)^{2,5}\right)^{0,4}\right\}$

$$
\begin{gathered}
(A \cup B)(x)=\min \{1 ; 0,6\} \\
(A \cup B)(x)=0,6
\end{gathered}
$$

Nessa etapa percebe-se a modificação no grau de pertinência, de acordo com a primeira e segunda simulações, de 1 para 0,6. Ao alterar uma das Falhas de Estrutura, que são paredes descascadas, além dos pesos para forma otimista, é possível obter melhora no resultado final com relação a Estrutura.

Quadro 24 - Limpeza coleta de resíduo

\begin{tabular}{|c|}
\hline Limpeza \\
\hline Complemento \\
\hline Coleta de resíduo $\quad$ W5 $=0,1$ \\
\hline 0,8 \\
\hline
\end{tabular}

Fonte: Autoria própria (2019)

Yager complemento: $A^{c}(\mathrm{x})=\left(1-0,8^{0,1}\right)^{10}$

$$
A^{c}(\mathrm{x})=0
$$

Quadro 25 - Limpeza

\begin{tabular}{|c|c|c|c|c|}
\hline \multicolumn{4}{|c|}{ Limpeza } \\
\hline Falta de \\
produto & $\begin{array}{c}\text { Produto de } \\
\text { baixa } \\
\text { qualidade }\end{array}$ & $\begin{array}{c}\text { Funcionários } \\
\text { sem } \\
\text { treinamento }\end{array}$ & $\begin{array}{c}\text { Falta de } \\
\text { cultura }\end{array}$ & $\begin{array}{c}\text { (NÃO) Coleta de } \\
\text { resíduo }\end{array}$ \\
\hline 0 & 0,6 & 0 & 0,7 & 0,01 \\
\hline
\end{tabular}

Fonte: Autoria própria (2019)

Yager interseção: $(A \cap B)(x)=1-\min \left\{1 ;\left((1-0)^{0,4}+(1-0,6)^{0,4}+\right.\right.$ $\left.\left.(1-0)^{0,4}+(1-0,7)^{0,4}+(1-0)^{0,4}\right)^{2,5}\right\}$

$$
\begin{gathered}
(A \cap B)(x)=1-\min \{1 ; 38,56\} \\
(A \cap B)(x)=1-1=0
\end{gathered}
$$

Quadro 26 - Má conservação do Campus Praça XI

\begin{tabular}{|c|c|c|}
\hline \multicolumn{2}{|c|}{ Má conservação do Campus Praça XI } & W1=5 \\
\hline Limpeza & Estrutura & Manutenção \\
\hline 0 & 1 & 1 \\
\hline
\end{tabular}

Fonte: Autoria própria (2019)

$$
\text { Yager União: } \left.(A \cup B)(x)=\min \left\{1 ;(0)^{5}+(0,6)^{5}+(0,86)^{5}\right)^{0,2}\right\}
$$


$(A \cup B)(x)=\min \{1 ; 0,89\}$

$(\mathrm{A} \cup \mathrm{B})(\mathrm{x})=0,89$

Com a alteração dos graus de pertinência e dos pesos foi possível obter resultado mais otimista com o grau de pertinência de 0,89 , ou seja, a alteração exposta na segunda simulação alcançou melhor desempenho para a Conservação do Campus, comparando-se com a situação atual do Campus.

\section{CONSIDERAÇÕES FINAIS}

Nesta seção será desenvolvida a estruturação da FDT utilizando como base a metodologia apresentada no item ÁRVORE DE DECISÃO FUZZY (FDT - FUZZY DECISION TREE), segundo os problemas de conservação observados no prédio que abriga o campus universitário, objeto do estudo de caso. Para tanto se faz necessário seguir os seguintes passos:

Quadro 27 - Análise detalhada dos resultados das simulações

\begin{tabular}{|c|c|c|}
\hline Situação Inicial & 1a Simulação & 2a Simulação \\
\hline \multicolumn{3}{|c|}{ Manutenção: Corredores - $(\mathrm{A} \cap \mathrm{B})(\mathrm{x})$} \\
\hline 0 & 0 & 0 \\
\hline \multicolumn{3}{|c|}{ 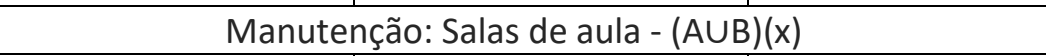 } \\
\hline 1 & 1 & 0,86 \\
\hline \multicolumn{3}{|c|}{ Manutenção: Banheiros - $(A \cup B)(x)$} \\
\hline 1 & 0 & 0 \\
\hline \multicolumn{3}{|c|}{ Manutenção - $(A \cup B)(x)$} \\
\hline 1 & 1 & 0,86 \\
\hline \multicolumn{3}{|c|}{ Estrutura - $(A \cup B)(x)$} \\
\hline 1 & 1 & 0,6 \\
\hline \multicolumn{3}{|c|}{ Limpeza: Complemento $-\mathrm{A}^{\wedge} \mathrm{c}(\mathrm{x})$} \\
\hline 0,01 & 0,01 & 0 \\
\hline \multicolumn{3}{|c|}{ Limpeza - $(A \cap B)(x)$} \\
\hline 0 & 0 & 0 \\
\hline \multicolumn{3}{|c|}{ Má conservação do Campus Praça XI - (AUB)(x) } \\
\hline 1 & 1 & 0,89 \\
\hline
\end{tabular}

Fonte: Autoria própria (2019)

Com base nas simulações realizadas e tendo em vista que para se ter melhora da condição de conservação do campus, o grau de pertinência final das falhas precisam se aproximar de 0 , foi possível identificar alterações significativas nos vários cenários, que são:

a) Manutenção - Salas de aula, com o grau de pertinência passando de 1 para 0,86 ;

b) Manutenção - Banheiros, onde o grau de pertinência foi alterado de 1 para 0 ;

c) Manutenção, tendo o seu grau de pertinência alterado de 1 para 0,86;

d) Estrutura, para o grau de pertinência alterado de 1 para 0,6; 
e) Limpeza - Complemento, como alteração do grau de pertinência de 0,01 para 0;

f) Evento Topo, Má conservação do campus, onde o grau de pertinência passou de 1 para 0,89.

Sendo assim, diante das três simulações, nota-se que é possível representar o contexto atual do campus, bem como a possibilidade futura de otimização dos recursos em geral para sua manutenção, associados à condição de análise do cenário de forma pessimista ou otimista, ratificando o uso da Árvore de Decisão Fuzzy para apoiar o gestor do campus em seu planejamento operacional e de manutenção.

Percebeu-se também que a diagramação da FDT facilitou a comunicação com pessoas que pertencem ao processo de planejamento, mas que não são técnicas em operação e manutenção.

Desta forma, considera-se que a FDT é uma técnica eficiente e de fácil utilização quando se têm diversas alternativas e cenários a serem analisados para um determinado problema (evento topo), apoiando o tomador de decisão na gestão, tanto no quesito planejamento quanto no de controle. 


\title{
Application of the fuzzy decision tree for conservation analysis of the private university campus
}

\begin{abstract}
Nowadays, property conservation is extremely important, because the good conditions of the environment interfere directly in the daily life of the place. Proper conservation avoids problems as property depreciation, accidents and structural problems. This study has the main objective to develop a case study of the university Estácio de Sá, on campus Praça X1, applying the concepts of Fault Tree Analysis (FTA) and the Fuzzy Decision Tree (FDT) to support the mapping of the causes of their poor conservation. After the FDT is structured, the quantitative analysis will be performed to create the simulation of the current scenario, as well as two more simulations for possible resources application, seeking for better results for the conservation of the Campus, optimizing the resources. To achieve this objective, applied, deductive, exploratory, qualitative and quantitative research is carried out to collect the data and necessary information to carry out the case study. The first simulation demonstrates the current condition of the Campus, considering a more pessimistic situation. The two other simulations were developed with the aim of improving the current situation of the Campus, considering a better allocation of the resources. For the first analysis, zero is used for the degree of pertinence of some causes, those considered important and of easy solution, maintaining the pessimistic vision. In the second simulation are maintained the pertinence degrees used in the first simulation, changing to an optimistic view.
\end{abstract}

KEYWORDS: Conservation, Fuzzy Decision Tree, Fault Tree Analysis, Cause and Effect Diagram. 
BANDO, Fernando Mucio; MARQUES, Jair Mendes; PATIAS, Josiele. Análise Probabilística de Árvore de Falhas na Gestão de Segurança de Barragens - Bloco D38 da Barragem de Itaipu. 2015. 14f. XXVI Seminário Nacional de Grandes Barragens. Foz do Iguaçu, 2015.

BARANAUSKAS, José Augusto. Funções Lógicas e Portas Lógicas. 2012. 105 slides. Departamento de Computação e Matemática - FFCLRP-USP. Disponível em: $<$ http://dcm.ffclrp.usp.br/ augusto/teaching/aba/AB-Funcoes-Logicas-PortasLogicas.pdf >. Acesso em: 20 de outubro de 2018.

BARROS, Jussara de. CUIDADOS COM A ESCOLA. s/d. Disponível em: $<$ https://educador.brasilescola.uol.com.br/gestao-educacional/cuidados-comescola.htm> Acesso em: 4 de agosto de 2018.

BENÍTEZ, César M. Vargas. FTA. s/d. 16 slides. Universidade Tecnológica Federal do Paraná - UTFPR.

CENTODUCATTE, Reinaldo; MACIEL, Ethel Leonor Noia; CAVALIERI, Edebrande; ALVES, Renato Carlos Schwab; GONÇALVES, Cinthya Andrade de Paiva; PRIMO, Francisco Caniçali; RAMOS, Maria da Penha; CASTRO, Marcio Chiabai Cupertino; MARTINS, Rosália Antunes; SANTOS, Anival Luiz; FANZERES, Ricardo da Silva; BRAZ, Erivelton Toretta; LAURINDO, Sebastião Marcelo; PEREIRA, Marcelo Rosa; TORRES, Fábio Magalhães; HERZOG, Gabriel da Silva; FILHO, Graciliano Alves da Costa. Manual de Execução dos Serviços de Limpeza: Empresa. 2015. Disponível em:

$<$ http://prefeitura.ufes.br/sites/prefeitura.ufes.br/files/field/anexo/manual_exec ucao_dos_servicos_de_limpeza_e_conservacao-empresa.pdf>. Acesso em 5 de agosto de 2018 .

Conheça a importância de ter uma gerência de manutenção predial. DGT Administradora de Condomínios. 2018. Disponível em: <https://www.dgtadm.com.br/importancia-gerencia-manutencao-predial/>. Acesso em 5 de agosto de 2018.

CONSERVAR. Dicionário do Aurélio. 2018. Disponível em: <https://dicionariodoaurelio.com/conservar>. Acesso em: 01 de Agosto de 2018.

DROZDA, Fabiano. Árvore de Falhas: Fault Tree Analysis - FTA. 30 slides. 2013.

ESPÍNDOLA, Rogério Pinto. Sistema Inteligente Para Classificação De Dados. 2004. 113f. Tese de Pós-graduação de Engenharia - Universidade Federal do Rio de Janeiro, COPPE. 2004. 
GOMIDE, Fernando A. C.; GUDWIN, Ricardo R.; TANSCHEIT, Ricardo. Conceitos Fundamentais da Teoria de Conjuntos Fuzzy, Lógica Fuzzy e Aplicações. 2018. 38f. Universidade Estadual de Campinas - UNICAMP. Disponível em: <http://www.cear.ufpb.br/juan/wp-content/uploads/2018/02/Artigo-Fuzzyconceitos-fundamentais.pdf $>$. Acesso em: 17 de novembro de 2018.

GUIA COMPLETO PARA LIMPEZA E CONSERVAÇÃO PREDIAL. MORHENA. 2017. Disponível em: <http://blog.morhena.com.br/guia-completo-para-limpeza-econservacao-predial/>. Acesso em 4 de agosto de 2018.

GUIMARÃES, Antônio C. F.; LAPA, Celso Marcelo F. Análise de Segurança em Centrais Nucleares Usando Lógica Difusa e Árvore de Falhas. Disponível em: < http://www.seeds.usp.br/pir/arquivos/congressos/CLAGTEE2003/Papers/TNPP\% 20B-167.pdf>. Acesso em: 30 setembro 2018.

LOPES, Mariana Vieira Ribeiro. Tratamento de imprecisão na geração de Árvores de Decisão. 2016. 100f. Universidade Federal de São Carlos - UFSCar. Programa de Pós - Graduação em ciência da computação. Disponível em: < https://repositorio.ufscar.br/bitstream/handle/ufscar/8954/DissMVRL.pdf?seque nce=1\&isAllowed=y>. Acesso em: 26 de Janeiro de 2019.

MAGRI, Juliana Mari. Aplicação do Método QFD no setor de serviços: Estudo de caso em um restaurante. 2009. 44f. Universidade Federal de Juiz de Fora. Disponível em: <http://www.ufjf.br/ep/files/2014/07/2009_1_Juliana.pdf>. Acesso em: 05 de janeiro de 2019.

MARRO, Alessandro Assi; SOUZA, Alyson Matheus de Carvalho; CAVALCANTE, Everton R. de Sousa; BEZERRA, Giuliana Silva; NUNES, Rômulo de Oliveira. Lógica Fuzzy: Conceitos e aplicações. s/d. 23f. Departamento de Informática e Matemática Aplicada (DIMAp). Universidade Federal do Rio Grande do Norte (UFRN) - Natal - RN - Brasil.

MORELLO, Marcelo Guimarães. Redução da árvore de falhas baseada no grau de criticidade: aplicação em transmissão de veículo comercial. 2005. $132 \mathrm{f}$. Dissertação de Pós-graduação - Universidade Estadual de Campinas, 2005.

MUNARO, Bianca; BORGES, Diogo; ARÊAS, Jonas; IGLESIAS, Renan; FARIAS, Vanius. Lógica Fuzzy: Introdução, motivação e conjuntos fuzzy. s/d. $36 \mathrm{f}$. Disponível em: <https://www.cos.ufrj.br/ mario/logica/logicaFuzzy.pdf>. Acesso em: 18 de novembro de 2018.

NETO, Odilon José de Oliveira; LIMA, José Jerônimo; CÉSAR, Vagner Antônio Máximo; REZENDE, Simone Oliveira; FIGUEIREDO, Reginaldo Santana. Aplicação do diagrama de causa e efeito e da análise swot na definição de prioridades em 
uma propriedade rural especializada em cria de bovinos. 2008. 19f. Disponível em: <https://ageconsearch.umn.edu/bitstream/108972/2/250.pdf>. Acesso em: 07 de Janeiro de 2019.

OTIMISMO. Dicionário Brasileiro da Língua Portuguesa. 2019. Disponível em: <http://michaelis.uol.com.br/busca?id=QwNGx>. Acesso em: 20 de Janeiro de 2019.

OTIMISMO. Dicionário do Aurélio. 2018. Disponível em:

<https://dicionariodoaurelio.com/otimismo . Acesso em: 20 de Janeiro de 2019.

OTIMISMO. Dicionário Online de Português. 2009. Disponível em:

<https://www.dicio.com.br/otimismo/>. Acesso em: 20 de Janeiro de 2019.

PESSIMISMO. Dicionário Brasileiro da Língua Portuguesa. 2019. Disponível em: $<$ http://michaelis.uol.com.br/busca? $\mathrm{r}=0 \& \mathrm{f}=0 \& \mathrm{t}=0$ \&palavra $=$ pessimismo $>$. Acesso em: 20 de Janeiro de 2019.

PESSIMISMO. Dicionário do Aurélio. 2018. Disponível em: $<$ https://dicionariodoaurelio.com/pessimismo . Acesso em: 20 de Janeiro de 2019.

PESSIMISMO. Dicionário Online de Português. 2009. Disponível em: <https://www.dicio.com.br/pessimismo/>. Acesso em: 20 de Janeiro de 2019.

PORTAL EDUCAÇÃO. Análise de árvore de falhas - AAF. s/d. Disponível em: < https://www.portaleducacao.com.br/conteudo/artigos/biologia/analise-dearvore-de-falhas-aaf/42909>. Acesso em: 21 de setembro de 2018.

PORTUGAL, Bruno Fragoso. Utilização de Árvore de Falhas como subsídio para manutenção centrada em confiabilidade de locomotivas. 2006. Monografia de conclusão da Especialização em Transporte Ferroviário de Cargas. Instituto militar de Engenharia. 2006. 65f. Disponível em:

$<$ http://transportes.ime.eb.br/etfc/monografias/MON005.pdf>. Acesso em: 07 de abril de 2019.

PRATI, Ronaldo. Inteligência Artificial: Aula Fuzzy. s/d. 38 slides. Disponível em: $<$ http://professor.ufabc.edu.br/ ronaldo.prati/InteligenciaArtificial/AulaFuzzy.pd f>. Acesso em: 17 de novembro de 2018.

PRODANOV, Cleber Cristiano; FREITAS, Ernani Cesar de. Metodologia do trabalho científico: Métodos e Técnicas da Pesquisa e do Trabalho Acadêmico. 2. ed. Rio Grande do Sul: Universidade Feevale, 2013. 
RIGNEL, Diego Gabril de Sousa; CHENCI, Gabriel Pupin; LUCAS, Carlos Alberto. Uma introdução a Lógica Fuzzy. 2011. 12f. Centro Universitário de Franca UniFACEF. Disponível em: <http://www.logicafuzzy.com.br/wpcontent/uploads/2013/04/uma_introducao_a_logica_fuzzy.pdf >. Acesso em: 17 de novembro de 2018.

SAKURADA, Eduardo Yuji. As técnicas de Análise dos Modos de Falhas e seus Efeitos e Análise da Árvore de Falhas no desenvolvimento e na avaliação de produtos. 2001. 145f. Dissertação de Pós-graduação - Universidade Federal de Santa Catarina, 2001.

SANTOS, Diônatas Simões dos; CECCATO, Maicon Silvio; MICHELON, Maikel Handerson. Eficiência da ferramenta 8D aplicada em uma indústria do setor Metal-Mecânico - Estudo de caso. 2011. 78f. FAE Centro Universitário. Disponível em: <http://img.fae.edu/galeria/getlmage/1/23567898199447012.pdf>. Acesso em: 05 de janeiro de 2019.

SCAPIN, Carlos Alberto. Análise Sistêmica de Falhas. 2. Ed. ISBN- 978-85-9825462-3, Nova Lima: Editora Falconi, 2013.

SILVA, Andréa de Benedetto. Operadores Booleanos: Como utilizá-los para recuperação de informações. 2016. Disponível em: <

http://biblioo.info/operadores-booleanos/>. Acesso em: 07 de outubro de 2018.

STONNER, Rodolfo. Análise de Árvore de Falhas - FTA. 2016. Disponível em: < https://blogtek.com.br/analise-de-arvore-de-falhas-fta/>. Acesso em: 23 de setembro de 2018.

SUCENA, Marcelo Prado. Curso de Especialização em Transporte Ferroviário de Carga - IME. Rio de Janeiro, 2008.

SUCENA, Marcelo Prado. Gerência da Manutenção: Análise de Risco. Rio de Janeiro, 2018.

SUCENA, Marcelo Prado. Métodos Matemáticos Aplicados à Engenharia de Produção/ Modelagem e Simulação de Sistemas: Teoria Fuzzy e Redes Neurais Artificiais. Rio de Janeiro, 2018.

WILGES, Beatriz; MATEUS, Gustavo Pereira; NASSAR, Silvia Modesto; BASTOS, Rogério Cid. Avaliação da aprendizagem por meio de lógica de fuzzy validado 
por uma Árvore de Decisão ID3. 2010. CINTED-UFRGS. Disponível em: <https://seer.ufrgs.br/renote/article/view/18053/10640>. Acesso em: 21 de outubro de 2018. crossref

XU, Dong; KELLER, James M; POPESCU, Mihail; BONDUGULA, Rajkumar. Applications of Fuzzy Logic In Bioinformatics. University of Missouri-Columbia, USA, Series on Advances in Bioinformatics and Computational Biology - Volume 9, ISBN-10 1-84816-258-8, Imperial College Press, 2008.

YAMANE, Alexandre Kenji; SOUZA, Luiz Gonzaga Mariano de. Aplicação do mapeamento de Árvore de Falhas (FTA) para melhoria contínua em uma empresa do setor automobilístico. 2007. Disponível em:

<http://www.abepro.org.br/biblioteca/enegep2007_tr580442_0041.pdf>. Acesso em: 07 de abril de 2019.

SUCENA, M.P. et al. Aplicação da árvore de decisão fuzzy para análise da conservação de campus universitário privado. R. Gest. Industr., Ponta Grossa, v. 16, n. 4, p. 161-185, Out./Dez. 2020. Disponível em: https://periodicos.utfpr.edu.br/revistagi

Correspondência:

Marcelo Prado Sucena

Instituto Militar de Engenharia (IME) - Rio de Janeiro, Rio de Janeiro, Brasil

Direito autoral: Este artigo está licenciado sob os termos da Licença Creative Commons-Atribuição 4.0

Internacional. 